\title{
JOURNAL OF THE ROYAL SOCIETY OF MEDICINE
}

\author{
December 2004 Volume 97 Number 12 ISSN 0141-0768
}

\section{Symbiogenesis}

We are now accustomed to the notion that an essential component of our cells, the mitochondrion, was originally derived from bacteria. Some two billion years ago, a singlecelled anaerobic life form was invaded by a rickettsia-like organism that survived from generation to generation and conferred the ability to metabolize oxygen. The 'serial endosymbiosis theory' says that the eukaryotic cell first came about through incorporation of free-living microbes of various sorts into a single more complex entity. Similar endosymbioses led to the origins of at least 26 phyla. With each new endosymbiosis, evolution took a leap forward - in the 'saltations' that were ill-explained by Darwin's theory of aggressive competition. For human genetics, this evolutionary story accounts for cytoplasmic inheritancewhereby genes in cytoplasmic inclusions can reproduce independently of those in the nucleus. Mitochondrial DNA, which is inherited solely from the mother, seems an important determinant of apoptosis and the speed of ageing. Various neurodegenerative diseases related to mitochondrial gene mutations are inherited maternally.

All this has been long been clear. Lately, however, the subject has moved forward with the proposal that retroviruses, as well as bacteria, contributed importantly to animal evolution. This is the subject of Dr Frank Ryan's article on the next page. About $8 \%$ of the human genome originates from retroviruses and if we measure viral products, almost half the human DNA is viral. Ryan reviews evidence that such retroviral components contributed both usefully and harmfully to mammalian life. On the positive side, a symbiosis of this sort may have led to development of the placenta, and certain key promoters, enhancers and silencers of genes owe their origin to these viruses. The negative side is that retroviral components have been linked to diseases including breast cancer, leukaemia, rheumatoid arthritis and multiple sclerosis; in some instances, the possibility of lowlevel transmissibility has been mooted.
Ryan suggests that the retroviral content of our genome probably reflects 'plague culling' - that is, a series of catastrophic epidemics in which only certain human genetic types survived, in mutually beneficial partnership with the viruses. There is no reason to think that this sort of evolution has come to a stop: the plagues probably arose when viruses crossed a species barrier, and as I write, a new retroviral transfer from ape to man is being reported from Africa. In addition, retroviruses may have contributed to evolutionary saltations by causing deletions, duplications and rearrangements of chromosomes. Small wonder that endogenous human retroviruses are attracting enormous attention: a casual inquirer on the Google search engine will find nearly 40000 entries.

A broader account of symbiosis and its contribution to evolution can be found in the book Darwin's Blind Spot ${ }^{1}$ in which Ryan expands on the notion of cooperation as fundamental to life on earth. I recommend it to anyone who is intrigued by his JRSM article. The story includes the more familiar phenomenon of 'exosymbiosis' in which different genera cooperate to their joint advantage - trees with fungi, fungi with ants, humans with gut bacteria. An optimist, Ryan reckons that human society is beginning to respond to its cooperative inheritance, evolving towards mutual help and away from ruthless competition. The concept links in with the Gaia hypothesis - the idea that our world is 'homeostatically controlled by active feedback processes operated automatically and unconsciously by the biota'. ${ }^{2}$ This is a comfortable notion. Another view is that, without some deliberate assistance from the human race, our planet will soon be in deep trouble.

\section{Robin Fox \\ Editor, JRSM}

\section{REFERENCES}

1 Ryan F. Darwin's Blind Spot: Evolution beyond Natural Selection. London: Texere/Thomson, 2003 [304 pp; ISBN 1-58799-115-2; £18.59]

2 Lovelock J. The Ages of Gaia. Oxford: Oxford University Press, 1988 